Materials and Methods Cluster random sampling in two states (Uttar Pradesh and Haryana) was used. A sample of 100000 people per district, with four districts per state was identified. Twenty per cent were selected from urban areas and rest from four randomly selected PHCs. In rural areas the survey was conducted with the help of ASHA workers and in Urban areas by the Urban Team. ASHA workers were provided with 2 days of comprehensive training and urban team was also trained.

Results We found that the total disability rate (TDR) was 67.3/ 10000 population-77.4/10 000 in males and 56.7/10 000 in females $(p<0.001)$. The TDR was significantly higher in urban areas than in rural areas $(83.2 / 10000$ vs $62.9 / 10000, \mathrm{p}<0.001)$. The same trend was observed at the state level. The data show that $58.2 \%$ of the total disability was due to locomotors defects only (polio, paralysis, epilepsy, congenital and trauma) followed by blindness and multiple disabilities. Further data will be available for the Congress.

\section{P1-205 A CASE CONTROL STUDY TO EVALUATE THE ASSOCIATION OF ARECA-NUT AND TOBACCO ABUSE AND ORAL PRECANCEROUS LESIONS IN NORTH INDIAN POPULATION}

doi:10.1136/jech.2011.142976d.98

S Kumar, ${ }^{*}$ D Mehrotra. King George's Medical University, Lucknow, Uttar Pradesh, India

Introduction Leukoplakia, erythroplakia, smoker's palate, and oral sub mucous fibrosis (OSMF) are potentially malignant precancerous conditions of the oral cavity which are thought to be intimately associated with the habit of chewing areca nut and tobacco either in the smoking or smokeless form.

Material and Methods A case control study was carried out in a representative North Indian population by organising oral health checkup camps. A validated questionnaire was administered to all subjects enrolled followed by their clinical examination. The suspected precancerous cases were confirmed by histopathological examination. Cases were defined on conformation of the biopsy report and matched with healthy controls. Univariate and multivariate analyses were performed and the ORs were calculated for the risk factors.

Results We found 448 cases of OSMF, 61 leukoplakia, 4 erythroplakia, 24 smokers palate and 17 oral lichen planus cases among 3136 subjects enrolled. Twelve cases confirmed as OSMF developed squamous cell carcinoma during the course of the study, however no such conversion is reported from other precancer groups till now. Strong correlation between the areca nut/tobacco products and oral precancerous lesions was observed. The OR ranged from 4.77 to 6.88 for areca nut users while for tobacco users it was 4.55-9.71, depending upon daily frequency of usage.

Conclusion The study revealed areca nut and tobacco as potential risk factors for the occurrence of oral precancer lesions.

\section{P1-206 EFFECTS OF CHILDHOOD SOCIOECONOMIC POSITION ON SUBJECTIVE HEALTH AND HEALTH BEHAVIOURS IN ADULTHOOD: HOW MUCH IS MEDIATED BY ADULT SOCIOECONOMIC POSITION?}

doi:10.1136/jech.2011.142976d.99

S Mckenzie, ${ }^{*} \mathrm{~K}$ Carter, T Blakely, V Ivory. University of Otago, Wellington, New Zealand

Introduction We examine the potential mediating role of multiple individual indicators of adult socioeconomic position (SEP) in the associations of childhood SEP with self-reported health measures and health behaviours.

Methods Data came from Wave 3 of the Survey of Family, Income and Employment. Childhood SEP was measured using parental occupation. Adult SEP was measured using education, household income, labour market activity and area deprivation. Self-rated health was assessed by a single-item measure. Psychological distress was assessed using the Kessler-10 scale. Current smoking status was derived from information on current and past cigarette use and binge drinking from information on the number of occasions of bingeing in the last 4 weeks. The associations of childhood SEP with each health outcome were determined using logistic regression, while adjusting for the mediating effects of individual adult SEP indicators.

Results Respondents from a lower childhood SEP had greater odds of being a current smoker, reporting poorer health and higher psychological distress. Two-thirds to three quarters of the association of childhood SEP with smoking (78\%), and psychological distress (66\%) and over half the association with selfrated health $(55 \%)$, were explained by educational attainment. Other adult socioeconomic indicators had much smaller mediating effects.

Conclusions The association between childhood SEP and self-rated health, psychological distress and current smoking in adulthood is largely explained through an indirect socioeconomic pathway involving education. Household income, area deprivation and labour market activity are still likely to be important as they are intermediaries in turn, in the socioeconomic pathway between education and health.

\section{P1-207 ASSESSMENT OF PERINATAL HEALTH SYSTEM AT THE POOREST REGIONS IN BRAZIL TO DECREASE INEQUALITIES IN INFANT MORTALITY}

doi:10.1136/jech.2011.142976d.100

${ }^{1,2}$ S Lansky, ${ }^{*}$ I H Perpetuo, ${ }^{1}$ E França, ${ }^{3} \mathrm{~S}$ Bittencourt, ${ }^{3} \mathrm{~S}$ Granado, ${ }^{3} \mathrm{M}$ do $\mathrm{C}$ Leal. ${ }^{1}$ Belo Horizonte Health Department, Belo Horizonte, Minas Gerais, Brazil; ${ }^{2}$ Federal University of Minas Gerais, Belo Horizonte, Minas Gerais, Brazil; ${ }^{3}$ National School of Public Health-Fiocruzuz, Rio de Janeiro, Brazil

Since 2008 Brazil is implementing a Project to decrease infant mortality in the Northeast and the Amazon Regions and reduce inequalities in these poorest regions of the country. An analysis of perinatal health indicators for all the 17 states and capitals that comprise these regions was conducted in order to support health planning and management for an effective perinatal public health system. Perinatal healthcare and population's health indicators were arranged in six groups: socioeconomics and demographics, primary healthcare (coverage, access and quality of contraception and prenatal care), hospital birth care, organization of perinatal public health system (referral system, transport, specialised care and neonatal intensive care units), quality of information on deaths and mortality rates. A perinatal health score system was assembled based on the states' and capitals' achievement in 21 and 18 variables, respectively. Each variable received 1 to 3 points to classify states and capitals in three levels: poor $(<33.3 \%)$, intermediate $(33.3 \%$ to $66.6 \%)$ and satisfactory ( $\geq 66.7 \%)$. Ten $(58.8 \%)$ states and four $(23.5 \%)$ capitals were classified into the "poor" group. Only 1 state and 6 capitals achieved the "satisfactory" group. "Organization of the perinatal public health system" and "infant and early neonatal mortality rates" were the groups with the lowest results for states and capital, as well as the "primary healthcare" group for capitals. This score system revealed which states and capitals must be prioritised in specific health actions to improve perinatal health, prevent avoidable infant deaths and decrease inequalities in the country. 